\title{
Design of Call Treatment System Based on MCU
}

\author{
Dandan Yan ${ }^{1, ~ a}$, Jinying Chen ${ }^{1, b^{*}}$, Shirui Huo ${ }^{1, c}$ and Tianmin $\mathrm{Li}^{1, \mathrm{~d}}$ \\ ${ }^{1}$ College of information science and technology, Chengdu University of Technology, Chengdu, \\ 610059, China
}

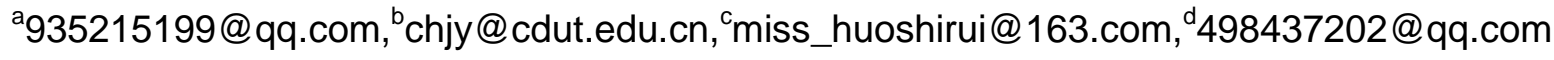

*The corresponding author

Keywords: Call treatment system; STC89C52; ISD1760; WiFi; Android; FPGA

\begin{abstract}
Since the calling range of current call treatment system is limited, resulting in staff retention in the waiting hall, noisy environment for treatment, work intensity of doctors and nurses, low treatment efficiency. A call treatment system based on MCU with STC89C52 MCU as the main control chip to control the ISD1760 voice broadcast, LED digital display and using RS232 serial port to form the call module. The smart phone is used as the receiving module to synchronously receive serial signals sent by WIFI module and voice information collected by FM chip .FPGA read the serial signals and convert them into VGA data, and then transmit it to the television display to form the display module. The patients who do not have smart phones or are not willing to install this APP can get the treatment process from the hospital's television screens or horns. Meanwhile, the patients who are on spot can synchronously check the treatment process through their smart phone. The results show that the system is stable and easy to operate. The call information can be conveyed to the waiting hall, and the Wi-Fi coverage area within a few hundred meters near the hospital.
\end{abstract}

\section{Introduction}

The reform in the medical system and the rapid development of the medical industry have promoted the Intelligentization of medical service [1]. Hospitals are improving the information construction by perfecting all kinds of medical services. Medical consultation is an important part of the medical service, and the phonetic call is the core link of treatment. Existing call treatment methods include manual calling and computer-based electronic calling system. In the human-based calling system, usually the nurse orally informs the patients to visit doctor according to the documents' orders submitted by patients [2]. Nowadays, the electronic queuing system based on computer is widely used. The calling part of the system includes LED screen which scroll displays the queue information of each consulting room, and the voice broadcast module which provides voice prompts when the LED display updates information [3]. From the calling part of the system we can see that the call system of the spreads information only to the waiting hall. Thus waiting patients have to stay in the waiting area and focus on the calling information. Although the electronic queuing system has alleviated the language and physical conflict caused by congestion, queue jumping, no orders and other instable factors incurred when people join the queue compared with the traditional mechanical human-based call system [4], the retention of patients in the waiting room can easily cause traffic congestion, especially in large hospitals, where the voice calling information is easily drowned in a noisy environment and the information of different consulting rooms scrolls on the same screen, which will confuse the waiting groups. This situation increases the work intensity of staff and shatters the waiting people. In view of the above problems in the call system, we have designed a call system based on the MCU. The calling information is transmitted by wireless communication, and displayed in several ways, reminding people through visual and audio devices. Calling information is issued by the doctor, and then communicated to the on-site and off-site patients in order to decrease the number of stranding citizens and solve the problem that people concentrate in waiting hall. 


\section{The Overall Design of the System}

The medical calling system based on MCU is composed of three parts including a calling module, a receiving module and a display module. The system structure diagram is shown in Fig. 1.The calling module is placed in every doctor's consulting room and doctors call patients through the buttons on panels. The display module assisted with a TV displayer is installed at the door of each room, displaying the calling information and informing those waiting patients of the process. The receiving module is realized by patients' mobile phone, which receives voice and text information synchronously.

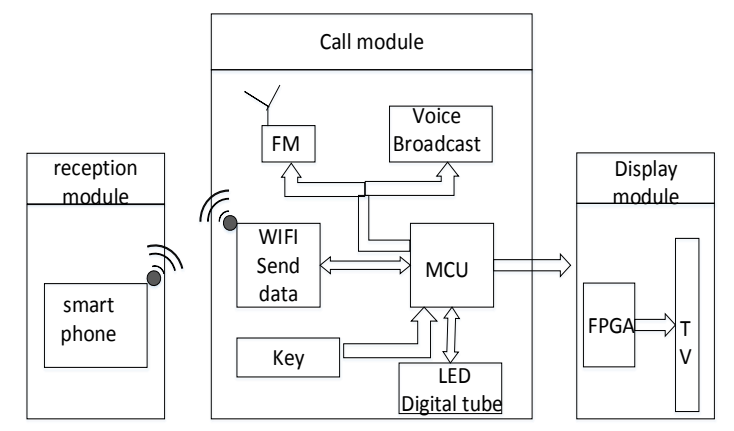

Figure 1. The system structure diagram

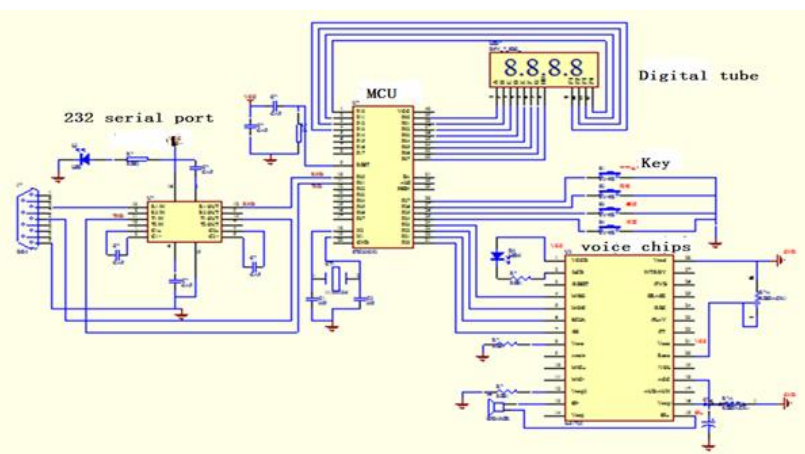

Figure 2. The circuit of call module

\section{Hardware Design of the System}

The system is divided into three parts including a calling module, receiving module and a display module. The information generated by calling is sent out through the WIFI module and FM chip, received by the smart phone, and sent to the TV screen to display through the serial port at the same time. The core circuit of the module is connected with the voice chip, keys, digital tube and serial port. The calling module is used to generate calling information and the buttons designed in call module are mainly used to generate calling information and save, recall, and clear calling information according to needs after operation.

\section{The Control Circuit of MCU}

The connection circuit of MCU, voice chip, keys, digital tube, and serial ports are shown in Fig. 2. In the Fig., the P2.0-P2.3 of STC89C52 is connected with SPI interface of the ISD1760 as a SPI connector, transmitting the address and data. The P3.0 receiver RXD and P3.1 transmitter TXD of the single chip are connected with the 9 pin serial port through the conversion chip MAX232 so as to realize long-distance transmission of data. P0.0-P0.7 is respectively connected with the segment selector A-DB of the digital tube. P1.0-P1.3 is respectively connected with the selected end P1-P4 of the digital tube so that SCM controls the digital tube displayer to present the call number. The P2.4-P2.7 of SCM is respectively connected with four keys to realize four functions such as call, memory, recall and reset.

\section{The Design of Voice Signal Generation and Dispatch}

MCU Controls the Broadcasting of a Voice Chip. ISD1700 series recording and playback chip can record several segment speeches. The sampling rate can be adjusted from $4 \mathrm{~K}$ to $12 \mathrm{~K}$ and the power is in the range of $2.4 \mathrm{~V}-5.5 \mathrm{~V}$. This design uses $5 \mathrm{~V}$ power of MCU to supply power, the voltage reaches $4.5 \mathrm{~V}$ when the system is operating, which meets the voltage range of a voice chip with the working voltage reaching $20 \mathrm{~mA}$ [5]. This design puts ISD1760 under the control of SPI mode.

Any four I/O pin of the master control chip AT89C52 is connected with the SCLK, MOSI, 
MISO, $\overline{S S}$ of the ISD1760 voice chip, which can control the subordinating voice chip to broadcast voice. When the $\overline{S S}$ bit is zero, the voice chip will open SPI interface as a controlled device, the SCLK connected to SPI interface of the master chip AT89C52 will control data input and output respectively on the MOSI and MISO of the voice chip. As the SCLK rises it is latched into the MOSI terminal of the voice chip along the data, the voice chip reads playback address from the MOSI. As SCLK falls, it will shift out from MISO terminal [6].

ISD1760 voice chip is needed to pre-insert with 12 sections of speech such as "please", "1"-"10", "visits". If the "calling" button starts with "01", the microcontroller sends " $01 "$ ", the voice chip reads playback address through the MOSI pin, which means the zero, first, and the twelfth speech will be invoked. Each voice segments is combined into a complete sentence and played as " No. 1, please ".At the same time, the voice chip sends back the current state of the voice chip to SCM through the MISO pin, in other words, whether the voice chip has received previous ISPI command and other information[7]。

FM Module Transmits Voice Signals .The T006+ FM wireless microphone module operates with the work frequency of $100.0 \mathrm{MHZ}$, voltage $3 \mathrm{~V}-5 \mathrm{~V}$ and the distance of $100 \mathrm{~m}$ in an open area (no obstacle between the transmitter and the receiver). Due to improving the voltage can increase the transmission distance, so this design uses $9 \mathrm{~V}$ voltage for power supply with the transmission distance up to 300-800 meters.

The voice broadcasted by voice chip for the doctor to confirm the calling information, and will be sent to the smart phone through the FM wireless microphone so that the calling information can be received in the absence of WIFI coverage.

The Microcontroller Sends Data to the Serial Port of RS232. Fig. 2 indicates, two data transmission channels are included. Long distance communication can be achieved between MCU and peripheral equipment's by using RS232 serial [8].Level conversion chip MAX232 converts TTL level into RS232 level, unifying levels on two parts so that no communication barriers come between them [9].

The serial port connected with WIFI module will send the data to smart phones. Simultaneously, FPGA module reuses this serial port, reads the serial port data for TV displaying.

\section{The Design of System Software}

The Software Design of the Calling Module. The main program detects the key and judge the key type. If it is the call button, the corresponding data will be sent to the P3-P4 of the digital tube to display and RS232 serial port will activate the voice chip to broadcast information. If it is the "storage" button is pressed, P1-P2 displays the same data of P3-P4. If the "recall" button is pressed, then perform a "call" button again with P1-P2 data while P1-P2 data will be reset .If it is the "reset" button, LED digital tube displays four full 0.This module includes key scanning program, voice calling program, digital tube display program, serial transmission program. The program flow chart is shown in Fig. 3.

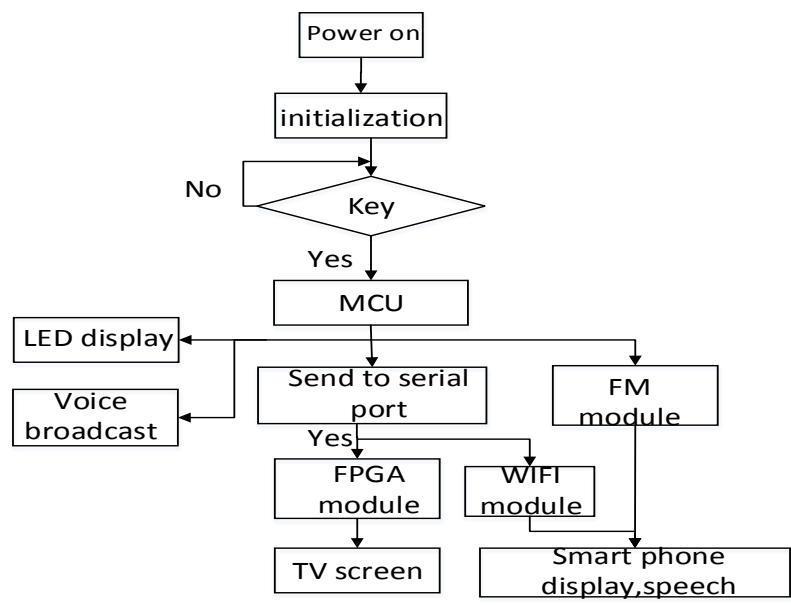

Figure 3. The program flow chart 
The Software Design of the Receiving Module. The Relink RT5350F chip is applied to develop a serial Wi-Fi module with M12L128168A chip added to provide system cache. The WIFI module is connected with the RS232 serial port to send serial data to the mobile phone in the form of wireless communication.

The Wi-Fi module as a server creates a socket, and is bounded to the specified address and port to monitor. When TCP connection initiated from the client mobile phone is monitored, it will establish a communication channel with the mobile phone. Wi-Fi module reads serial data and sends to the mobile phone. The intelligent mobile phone as a client to connect to the specified Wi-Fi network and displays the schedule designed based on Java and Android technology on the APP screen [10], which applies to smart phones with Android system so that patients can check the queue schedule. The software can display the calling information in written form.

The Software Design of the Display Module. FPGA contains four modules: VGA timing generator module, VGA image display palette module, data storage and data read-write controller. VGA timing generator module generates the timing signal required by the displayer including timing and field sequence, which is the key to complete the design since timing accuracy is necessary for correct display. Data storage unit and data reading and writing controller solve the source of display data and data storage.

The data reading and writing controller in FPGA reads the RS232 serial data and IP kernel ROM and RAM store it. The data is YUV signal, which is converted into RGB signal by the VGA image display module palette, then transferred to the VGA interface chip ADV7125.The VGA interface receives the horizontal sync signal, vertical synchronous signal and $R, G, B$ three kinds of color signal $[11,12]$. The queues check the display information outside the consulting room to accurately grasp the queue schedule.

\section{Conclusion}

After the software testing, hardware testing and system debugging, the system can achieve the expected function of sequential calls, recall, visiting number reset, visiting number display, cell phone display with treatment schedule, and the radio voice broadcast schedule. The loud speaker is sonorous; the digital tube displays normally; Wi-Fi data is received and transmitted stably; the central processor MCU and mobile phone procedures operate normally. The double function of wireless transmission of texts and language is realized. The disadvantage is that voice synchronization on radio broadcasting presents slightly distorted signal with interference of noise. The results show that the calling system can reduce the waiting time of patients, standardized treatment process, improve the quality of nursing service and patient satisfaction. To a certain extent, it can achieve long distance wireless call and solve the problems of the existing calling system. At the same time, the system can also be applied in other occasions of queue, such as bank queuing for business, restaurant for taking meals, and so on.

\section{Acknowledgements}

This work presented in this paper is supported by the Huimin project of Chengdu city science and technology (2014-HM01-00160-SF); The National College Students' innovation and entrepreneurship training programs (201510616092)

\section{References}

[1] Qian Hao, Jiang Lin, Li Lizhen, et al. The design of ward call system based on MCU [J].Computer Technology and Development, 2014, 24 (3):210-213.

[2] Zhang Xinyue; Hospital queuing system based on Andrews [J].China science and technology information, 2014, (6):148-149. 
[3] Song Zhihui, Wei Pingping, Guo Zhenming; The overview of Application status of hospital queue queuing system [J]. The computer age, 2012, (11):10-11.

[4] Soheila Mojdeh,Mohammad Mehdi Nadi,Kasra Gordiz,et al.Especial nurse call system for ICU patient—Nurse call system[J] Journal of Biomedical Science and Engineering, 2013, Vol.06 (08), pp.843-846.

[5] Anonymous, Sound makes chips [J],Environmental Engineering, 2014, Vol.27 (4)

[6] [Feng Zhihui, Xu Lijun, Xue Pengtao, et al.. A single-chip microcomputer control circuit based on ISD4004 voice chip application [J]. Control \& Automation, 2003, 19 (7).

[7] Huo Lin, Yan Wenyuan; Design and implementation of the system call number [J].Journal of Guangxi Academy of Sciences, 1999,15 (1):26-28.

[8] Shen Kai, Wu Qingping; Ward call system design based on MCU [J]. Modern Electronics Technique, 2011, 34 (1):141-142.

[9] [Magda, Yury, Serial port tests digital circuits [J], Test \& Measurement World, 2009, Vol.29 (3), pp.24.

[10]Domenico Amalfitano,Anna Rita Fasolino,Porfirio Tramontana,et al. Android Mobile Applications: Challenges, Strategies, and Approaches [J], Advances In Computers, 2013, Vol.89.

[11]Chen Jinying. FPGA technology and Application [M]. Beijing: Machinery Industry Press. 2015.3

[12]Zhang Yaping, Zhan Zhuang He. Module design of FPGA display based on VGA [J].Computer Technology and Development.2007, 17 (6): 242-245. 